Musées, Patrimoine et Culture scientifiques et techniques

$150 \mid 2013$

novembre-décembre 2013

\title{
Éléments de prospective muséale
}

François Mairesse

URL : http://journals.openedition.org/ocim/1294

DOI : 10.4000/ocim.1294

ISSN : 2108-646X

Éditeur

OCIM

Édition imprimée

Date de publication : 29 novembre 2013

ISSN : 0994-1908

\section{Référence électronique}

François Mairesse, «Éléments de prospective muséale », La Lettre de l'OCIM [En ligne], 150 | 2013, mis en ligne le 29 novembre 2015, consulté le 19 avril 2019. URL : http://journals.openedition.org/ ocim/1294; DOI : 10.4000/ocim.1294

Ce document a été généré automatiquement le 19 avril 2019

Tous droits réservés 


\title{
Éléments de prospective muséale
}

\author{
François Mairesse
}

1 Les fins de siècles et les débuts de millénaires apparaissent comme des moments propices pour s'interroger sur le futur des musées; les temps de crise également. La conjonction des deux phénomènes a donné lieu à la publication d'un grand nombre d'ouvrages et de rapports questionnant ou tentant de prédire l'avenir de l'institution. Le substantif féminin "prospective », qui remonte aux années 1950, est utilisé pour définir l'approche interdisciplinaire liée à l'étude des mutations d'un secteur de la société ou d'une institution. Ce concept diffère de celui de prévision dont la temporalité est plus courte, mais il est parfois associé à celui de futurologie, qui peut embrasser un horizon temporel plus lointain. Les recherches prospectives évoquées ici ne s'intéressent pas au musée de 2015 ou à celui de 2100, mais plutôt à celui de 2025 ou de 2030 : une quinzaine ou une vingtaine d'années durant lesquelles nombre de changements pourraient se produire, sans pour autant envisager de bouleversements radicaux (la production en série de soucoupes volantes ou le clonage humain). Les méthodes de la prospective, en effet, diffèrent sensiblement des approches "visionnaires ", plus ou moins farfelues, se fondant sur une méthode rigoureuse incluant l'étude des grandes tendances pouvant influencer l'institution - démographie, technologie, éducation, économie... - et visant à élaborer plusieurs scénarios à partir de ces futurs. Ces scénarios constituent chacun un possible avenir du secteur étudié, les experts s'accordant sur le fait que le futur devrait très vraisemblablement apparaître comme un mixte de ces différents scénarios. Ainsi, par exemple, le National Intelligence Council publie régulièrement des rapports sur l'état du monde dans une quinzaine d'années ${ }^{1}$. De même, le ministère de la Culture a récemment commissionné une étude envisageant la politique culturelle en $2030^{2}$. 


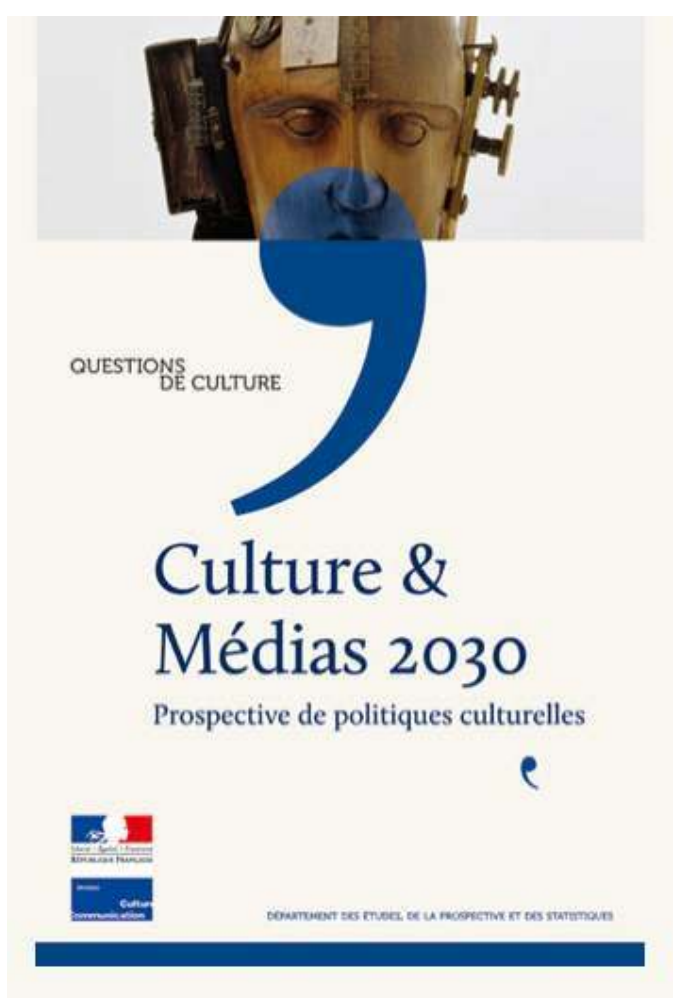

Une étude sur la politique culturelle en France à I'horizon 2030

(c) DR

2 Si l'on trouve des articles ou des ouvrages abordant le futur des musées depuis la fin du $\mathrm{XIX}^{\mathrm{e}}$ siècle, c'est essentiellement à partir des années 1970 que l'on peut constater un réel intérêt pour le futur de l'institution, peut-être justement parce qu'elle entre en crise, tant pour ce qui concerne sa nature profonde (qui donnera naissance, en France, au mouvement des écomusées) que pour ses modes de financement (le choc des années 1973-1974 et les changements de politique économique qui en résultent). Ce mouvement de réflexion ne va jamais réellement disparaître: depuis ce moment, plus d'une cinquantaine d'ouvrages et de rapports (plus de 5500 pages) ont été produits sur le futur du monde muséal, le phénomène s'accélérant avec le tournant du millénaire (avec des titres comme L'avenir des musées, Les musées et le nouveau millénaire, La transformation des musées au $\mathrm{XXI}^{\mathrm{e}}$ siècle...). Dans la plupart des cas, il ne s'agit pas, à proprement parler, de réflexion prospective, mais plutôt d'analyse des tendances ou des réflexions les plus innovantes semblant se dégager du monde des musées contemporains.

La crise économique, qui embrase le monde occidental depuis l'éclatement de la bulle spéculative de 2007, renforce l'incertitude envers l'avenir des organisations culturelles. De nombreux musées sont touchés par les mesures de restrictions budgétaires qui en découlent (dans les pays méditerranéens, mais aussi en Grande-Bretagne ou aux ÉtatsUnis), certains se voyant contraints de fermer définitivement leurs portes. C'est dans ce contexte qu'ont été publiés plusieurs rapports anglo-saxons explorant le futur des musées, sur lesquels il peut sembler intéressant de s'attarder quelques instants. 


\section{Le Center for the future of Museums et les associations de musée}

Le rôle joué par les associations de musées dans l'élaboration de ces rapports prospectifs est à cet égard particulièrement important. On notera, en France, l'implication des différentes associations autour du Livre blanc des musées de France, publié en 2010 dans la revue Musées et collections publiques de France, qui tout en n'étant pas directement tourné explicitement sur le futur muséal, s'interroge sur l'avenir de l'institution. L'American Alliance of Museums, dans cette perspective, s'est inscrite à la pointe de ce mouvement de réflexion en créant, en 2008, le Center for the future of Museums qui développe une activité éditrice très soutenue depuis sa fondation. Le centre a décidé de se consacrer principalement à l'étude de trois tendances à ses yeux fondamentales pour comprendre le futur des musées: la démographie, l'éducation et les technologies numériques mobiles. Pas moins de six rapports et un très grand nombre de notes ou de vidéos ont ainsi été publiés afin de guider les musées dans leur réflexion prospective. Car si l'association se charge de cette mission, c'est dans une perspective d'évolution et d'adaptation du monde des musées, afin de permettre à chaque établissement de se préparer aux mutations qui, immanquablement, devraient arriver dans les années à venir. Un tel exercice apparaît comme relativement courant dans le monde des entreprises et de la réflexion stratégique, aussi n'est-il guère étonnant de voir la prospective se développer dans les pays les plus en pointe en matière d'intégration des règles du management dans les musées.

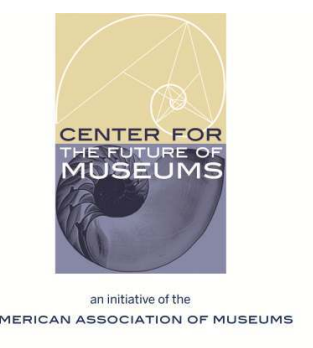

MUSEUMS \& SOCIETY 2034: TRENDS AND POTENTIAL FUTURES

VERSION 1.0

DECEMBER 2008

Un rapport sur l'évolution des musées américains en 2034, réalisé par le Center for the future of Museums mis en place en 2008 par l'American Alliance of Museums.

(C) DR 
C'est une logique similaire que l'on retrouve dans deux autres associations de musées : la Museums Association britannique et l'association des musées aux Pays-Bas. Sans doute ne sera-t-on pas étonné de retrouver cette même démarche dans les associations de ces deux pays partageant avec les États-Unis une vision assez similaire de la gouvernance publique et de la gestion muséale qui en résulte. Quoi qu'il en soit, les musées hollandais envisagent à leur tour, en 2010, un travail prospectif à partir de leur contexte national, et c'est au tour des Britanniques, deux années plus tard, de lancer un mouvement de consultation sur le futur des musées. Chacune de ces études semble partir d'un constat identique : le monde des musées a connu, durant les dernières décennies, une période de transformations considérable, voyant leur nombre doubler et leur popularité s'accroître. Il est probable que ces années aient constitué, en quelque sorte, un âge d'or pour les musées; celle des années 2010 pourrait, au mieux, se présenter comme une période de consolidation, mais peut-être aussi de décroissance. Des voix s'élèvent ainsi, notamment en Allemagne, pour suggérer de réduire de moitié le nombre d'établissements culturels subventionnés par l'État.

6 Je voudrais évoquer ici les résultats présentés dans six rapports ou ouvrages, à mon sens assez représentatifs de ce type de littérature : trois ont été rédigés par le Center for the future of Museums. Il s'agit du premier des rapports par lequel le centre a inauguré en 2008 sa politique éditoriale, Museums and society 2034 : trends and potential future, ainsi que de deux rapports plus récents intitulés Trendswatch 2012 et Trendswatch 2013, décrivant chacun un certain nombre de facteurs supposés influencer le monde des musées dans l'avenir ${ }^{3}$. L'association hollandaise des musées, quant à elle, s'est penchée sur l'avenir de son secteur avec l'Agenda 2026. Study on the Future of the Dutch Museum Sector ${ }^{4}$. L'un des derniers rapports en date, dont le processus de rédaction n'est pas tout à fait terminé à l'heure où ces lignes sont écrites, est produit par la Museums Association. Il s'agit du document de discussion Museums 2020, présentant le possible avenir des musées britanniques et qui a été soumis à un large processus de discussion au sein de la communauté muséale ${ }^{5}$. Enfin, un dernier ouvrage, rédigé par un couple de muséologues hollandais, Peter et Léontine Van Mensch, analyse les nouvelles tendances dans le domaine de la muséologie et des musées ${ }^{6}$. La vision présentée par ces différents documents n'est pas pour autant revendiquée comme nationale. Certes, chaque pays présente des particularités qui se retrouvent dans les rapports. Ainsi, les États-Unis insistent sur leur population multiethnique, les Pays-Bas évoquent leur conurbation (le Randstad) ainsi que l'influence de l'Union européenne, tandis que la Grande-Bretagne se penche sur son système de financement ${ }^{7}$. Il n'en demeure pas moins que si aucun de ces rapports ne prétend à une vision universelle, leur lecture conjointe permet de faire apparaître un certain nombre de récurrences. En dernier lieu, la nature de l'ouvrage des Van Mensch diffère de celle des rapports rédigés par les associations, leur réflexion se voulant plus théorique, abordant certaines questions liées au champ muséal dans son ensemble.

\section{Quelles évolutions du musée pour le futur?}

Globalement, la plupart des rapports se fondent sur des facteurs identiques - et guère originaux - pour étudier les transformations du monde muséal.

1/ La démographie constitue un premier élément d'importance, et surtout le vieillissement des populations, mais aussi les transformations ethniques de certains pays 
(notamment aux États-Unis) entraînant nombre de conséquences importantes pour les années à venir. Ainsi, les musées devraient songer, dans les années futures, à traduire plus souvent leurs textes, augmenter la taille des caractères des panneaux explicatifs, prévoir plus de sièges ou plus d'accessibilité pour les chaises roulantes, positionner le musée comme lieu d'entretien des facultés intellectuelles... Par ailleurs, les transformations démographiques risquent de renforcer l'augmentation des écarts de revenus entre différents groupes de la population.

2/ Un phénomène qui est directement associé à la démographie, mais qui regroupe également d'autres facteurs, est celui de la mondialisation, et notamment ses influences sur l'urbanisme et le tourisme mondial, ces deux facteurs jouant un rôle décisif dans le fonctionnement de certains musées actuels - comme le montre l'exemple du musée Guggenheim de Bilbao, si souvent cité. Ici aussi, l'augmentation du tourisme mondial, telle qu'elle est pressentie, nécessitera des adaptations vers les nouveaux groupes de touristes (en provenance d'Asie ou d'Amérique du Sud). Mais la mondialisation va également entraîner la poursuite du développement des mégalopoles, induisant une augmentation du prix des logements et la réduction des surfaces d'habitation. Les musées pourraient, dans cette perspective, renforcer leur attractivité en développant leur fonction de lieu public, au gré de la diminution de l'espace privé.

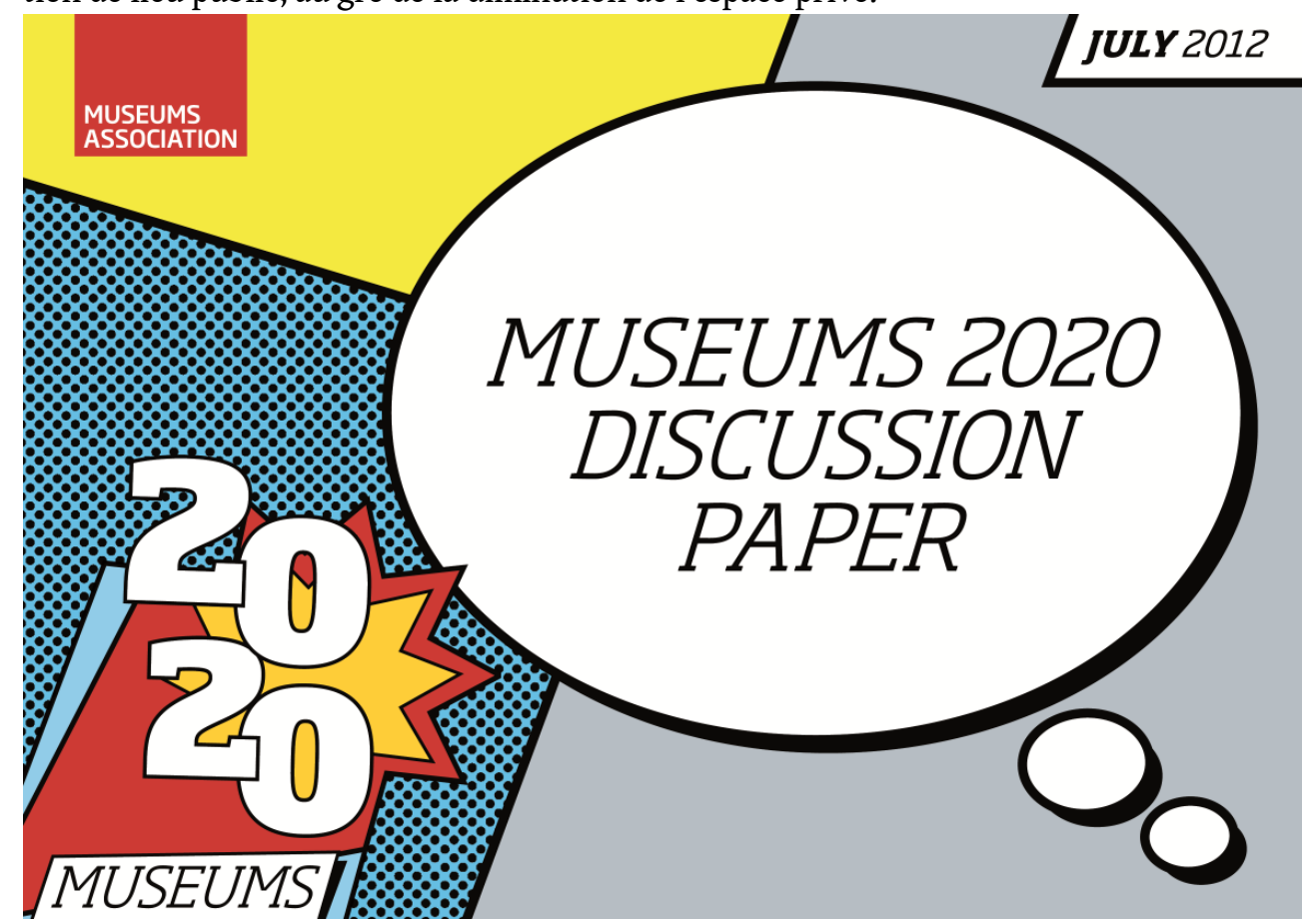

L'avenir des musées britanniques en 2020 vu par la Museums Association

(C) DR

3/ L'analyse de l'activité économique constitue un autre pilier d'analyse de toutes les enquêtes. Ce volet peut être envisagé de diverses manières, qu'il s'agisse des changements de politiques économiques (l'analyse des subventions) ou de l'étude du prix de l'énergie et de son influence sur les coûts des transports, notamment en regard de la fréquentation des visiteurs. L'exploration de nouvelles techniques de financement ou de nouvelles manières de penser l'économie (comme l'économie de l'expérience ou celle de la créativité) constitue également une manière de penser le positionnement des musées face à ces transformations. 
4/ L'innovation technologique, notamment dans le domaine du numérique, est évoquée depuis longtemps comme une source de transformation des musées. La numérisation du patrimoine, l'avènement des imprimantes 3D et de la réalité augmentée sont perçus comme autant de facteurs pouvant transformer les musées et la manière dont le public interagit avec eux. Par-delà la numérisation ou l'avènement d'innovations technologiques dont les musées constituent partiellement la vitrine, d'autres aspects peuvent être évoqués, comme par exemple la possibilité de présenter ces établissements comme des lieux "non connectés", havres de quiétude au sein d'un univers en perpétuelle sollicitation numérique.

5/ L'évolution de l'éducation apparaît également (notamment pour les Américains) comme un facteur décisif de la transformation du monde muséal. Le rôle éducatif des musées ne date pas d'hier, mais la logique de l'auto-apprentissage (free choice learning), mise en valeur par John Falk et Lynn Dierking, notamment, est souvent présentée comme une opportunité pour les musées face à l'obsolescence de la formation initiale traditionnelle (tant pour l'école que pour l'université). L'apprentissage « tout au long de la vie » n'a jamais été plus souvent plébiscité, et les programmes mis en place par les musées pourraient participer à la logique de micro-curriculums alternatifs dont l'agrégation pourrait, à terme, être validée sous une forme ou une autre (des expériences existent déjà à ce sujet aux États-Unis).

6/ Enfin (ce domaine est surtout analysé par les Van Mensch), la technique muséale ellemême peut évoluer du fait de ses particularités propres, et notamment ses collections, mais aussi ses interactions avec les autres institutions du patrimoine, telles que bibliothèques et centres d'archive.

C'est à partir de ce faisceau d'analyses que trois types de répercussions sont particulièrement mis en lumière: la retraite des boomers, la récession et le crowdsourcing. La mise à la retraite progressive de la génération du baby boom, née juste après la Seconde guerre mondiale, constitue en effet un changement important. Cette génération, qui a vécu pleinement les années de croissance de l'après-guerre, bénéficie d'un pouvoir d'achat important et d'une espérance de vie supérieure à celle des générations précédentes. Plusieurs auteurs se réjouissent de l'augmentation du temps libre dont disposera cette génération, lequel pourrait être utilisé au bénéfice d'une pratique plus assidue des musées ou dans le cadre d'un investissement bénévole. En même temps, il n'est pas impossible que ces jeunes retraités se retournent vers d'autres projets qui leur sont également accessibles, par exemple les voyages à l'étranger. Si cette génération dispose d'une plus grande espérance de vie et d'un bon pouvoir d'achat, il n'en reste pas moins que les musées vont devoir s'adapter à de potentiels visiteurs plus âgés (et exigeants). 


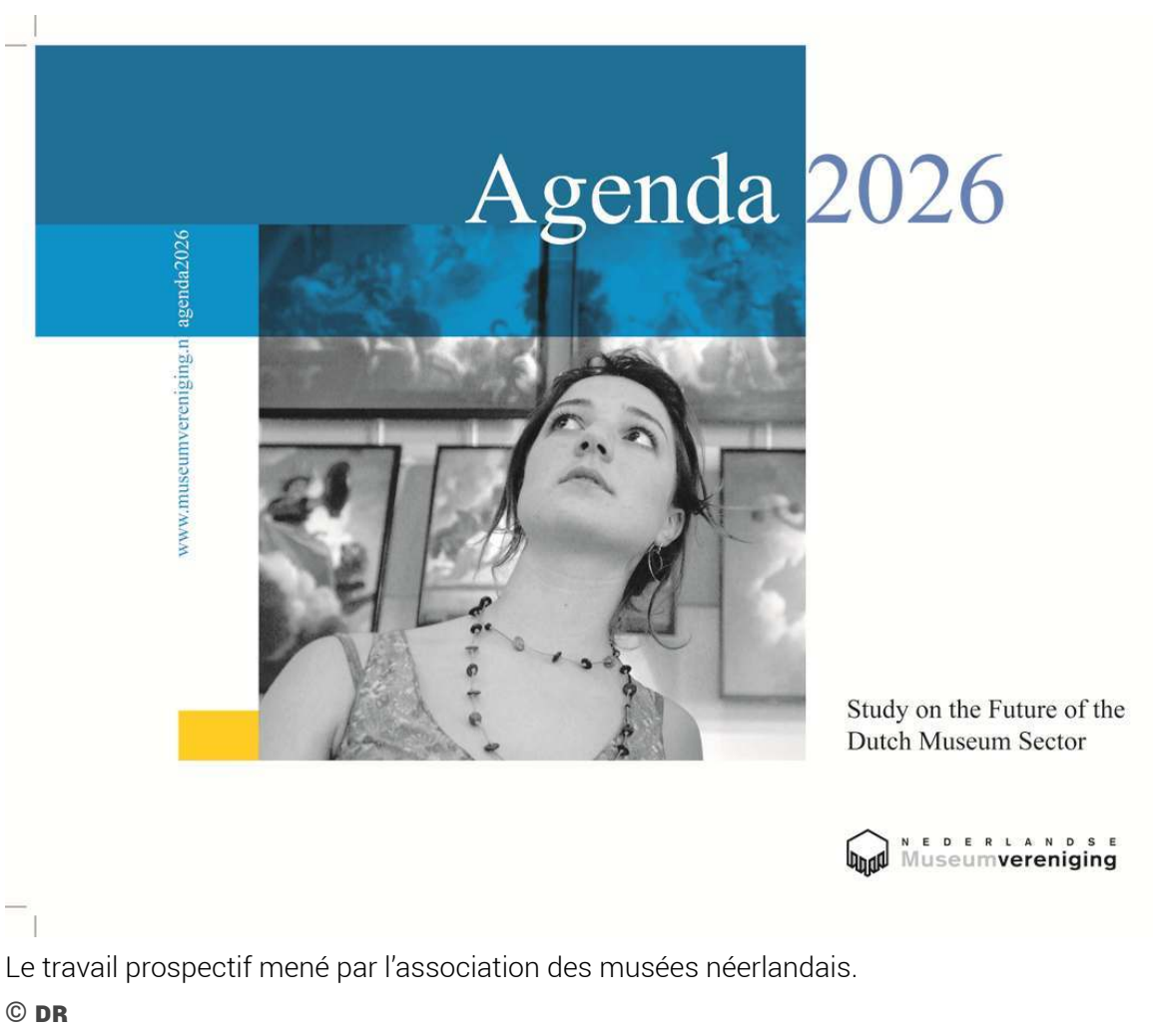

8 La crise économique constitue un autre élément régulièrement évoqué. De manière spontanée, la plupart des rapports - autant aux États-Unis qu'en Grande-Bretagne ou aux Pays-Bas - considèrent la baisse structurelle des subventions publiques comme acquise. Par ailleurs, suggèrent plusieurs auteurs, le risque de persistance de la crise économique induira une diminution du pouvoir d'achat d'une partie de la population, et probablement l'augmentation des écarts de revenus au sein de la société. Dans un tel contexte, les musées doivent se mettre à la recherche de nouveaux modèles économiques, reposant moins sur les pouvoirs publics, sans pour autant s'appuyer sur une logique trop commerciale. L'une des solutions les plus régulièrement présentées est liée aux nouvelles technologies, à la révolution 2.0 et au musée participatif. Cette logique, qui a fait l'objet de nombreux articles et ouvrages (le plus classique étant The participatory Museum de Nina Simon), touche aussi bien au financement par la foule (crowdfunding) qu'au travail bénévole et, de manière plus générale, à la participation de la population dans la gestion muséale (crowdsourcing). Une telle logique, déjà largement appliquée de manière plus ou moins artisanale dans nombre de musées associatifs, induit des changements significatifs dans la gestion même de l'institution, puisque la relation «pro-am» (professionnelamateur) transforme, en quelque sorte, les rapports de production - des amateurs pouvant par exemple participer à la conception d'expositions, ou à des recherches - et donc à la production elle-même, soit au contenu du musée et à l'éthique de son fonctionnement.

\section{Une tendance qui s'impose?}

Mis à part l'ouvrage des Van Mensch, les rapports insistent peu sur l'évolution interne du secteur muséal lui-même, notamment les questions plus spécifiquement liées aux collections, et par exemple les nouvelles manières dont celles-ci pourraient être utilisées 
dans les années à venir. Le principe de la mobilité des collections, visant à mieux les faire circuler et ainsi favoriser leur utilisation, avait fait l'objet de nombreuses études dès le début des années 2000. Le lien entre les objets et les technologies numériques ne passe pas non plus seulement par leur dématérialisation, mais aussi par l'Internet des objets (souvent présenté, à l'instar du web sémantique, comme une troisième révolution de la toile), à savoir la possibilité pour chaque objet d'être connecté et de livrer ses informations. On peut être étonné, par ailleurs, de ne pas voir détaillées les transformations du paysage muséal dans son ensemble. À cet égard, on pourrait notamment évoquer la constitution d'une classe supérieure de très grands musées (les "musées superstars", millionnaires en visiteurs) dont les modes de fonctionnement diffèrent de plus en plus de ceux des autres établissements, s'inscrivant progressivement dans une logique identique à celle des industries culturelles.

En revanche, et sans doute en dehors de ces très grands «musées superstars », la tendance principale du monde muséal qui semble s'imposer repose sur ce lien de plus en plus fort entre le musée et sa communauté - ou plus précisément la community (dans le sens des parties prenantes ou stakeholders, intégrant publics, spécialistes, journalistes locaux, autres lieux de conservation ou de recherche...). L'exemple le plus frappant de cette logique se trouve sans doute dans le rapport britannique Museums 2020, dont la structure repose sur l'articulation du musée avec l'individu, la community, la société et l'environnement. La relation entre le musée et son territoire est ancienne, on la retrouve par exemple déjà dans les travaux de Brown Goode, à la fin du XIXe siècle. Pendant longtemps, pourrait-on dire, c'est cependant plutôt la manière pour le musée de s'adapter à l'économie de marché qui avait été présentée comme primordiale, le modèle de Bilbao constituant, en quelque sorte, l'un des jalons forts de cette logique économique. La sortie d'ouvrages sur le travail social du musée ou sur les questions d'inclusion sociale, montre une tendance que certains auteurs, comme Black, évoquent comme la plus importante pour les années à venir ${ }^{8}$.

Le rôle social des musées constitue cependant également un fait ancien, dont l'histoire remonte au moins au XIX ${ }^{\mathrm{e}}$ siècle. Ce phénomène a connu des évolutions importantes, et s'il a notamment été en vogue durant les années 1970-1980, à travers la nouvelle muséologie en France ou le concept de musée de voisinage aux États-Unis, il avait connu un succès nettement plus relatif dans les décennies qui suivirent, alors que le spectaculaire muséal s'imposait au gré des nouvelles constructions architecturales remarquables et de l'engouement pour les grandes expositions populaires. C'est peut-être pourtant une sorte de mouvement cyclique qu'il convient de reconnaitre dans l'évolution actuelle, alors que la question du lien social ressurgit de plus belle au début du XXI siècle, notamment à travers les réflexions de Richard Sandell sur l'inclusion sociale. 


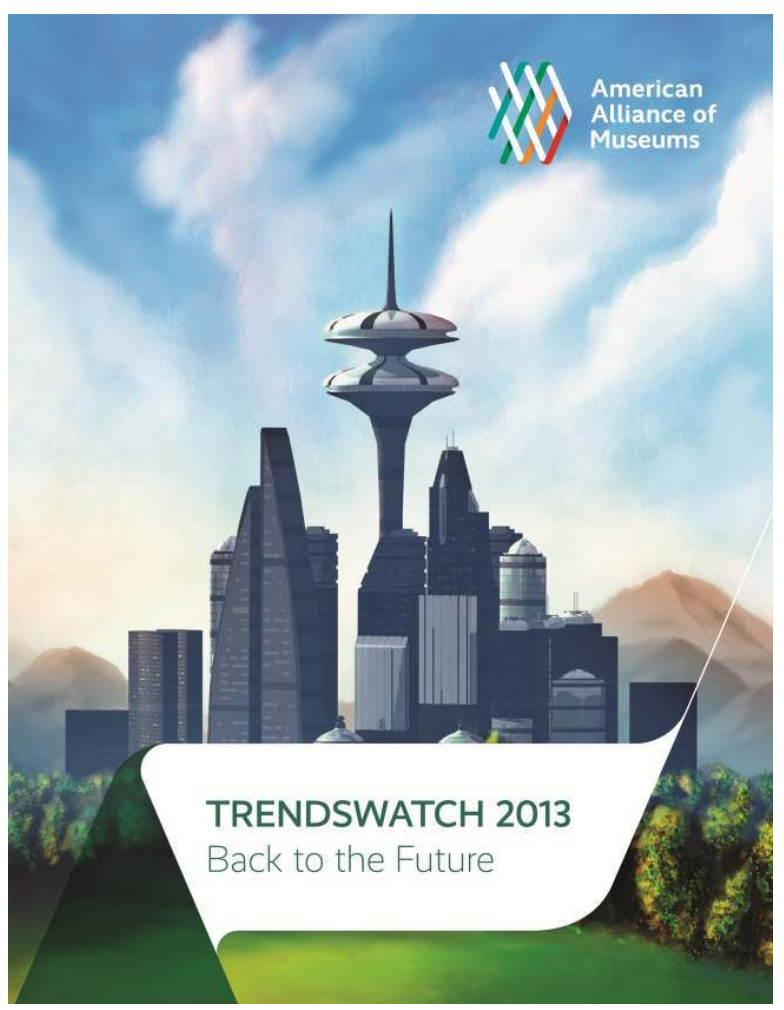

Trendswatch 2013, édité par l'American Alliance of Museums, se penche sur les facteurs qui influenceront le monde des musées dans le futur.

(c) DR

On pourrait conclure, au vu de ces quelques informations, que tous ces facteurs évoqués semblent bien familiers et que le futur du monde des musées, tel qu'il se présente ici, ne diffère pas sensiblement de la situation que nous connaissons de nos jours. Le contraire eut été suspect : la prospective n'est pas la science-fiction, elle se fonde sur des données simples à partir desquelles nous pouvons déterminer un certain nombre de tendances relativement prévisibles. Il n'est cependant pas impossible que notre futur soit transformé de manière radicale par une innovation technologique (comme a pu l'être Internet) ou par un bouleversement politique (comme l'effondrement du système soviétique). Pour autant, si l'on peut aisément concevoir l'influence de la démographie, de l'économie, des technologies numériques ou de la mondialisation, force est de reconnaître qu'il est rare de lire, peut-être avec autant d'assurance, que les subventions publiques sont irrémédiablement amenées à diminuer et qu'il devient impératif de se tourner vers d'autres modèles de financement - notamment le modèle participatif.

Ainsi, de nombreuses études semblent montrer que le futur du musée sera social ou ne sera pas, et que pour survivre, l'institution se devra d'intégrer en premier chef ce lien qui la relie avec son milieu social. Un tel scénario, pour plausible qu'il soit, ne doit pas oblitérer le fait que le paysage muséal demeure complexe et qu'il n'existe pas un mais plusieurs futurs pour les musées, selon qu'ils soient grands ou petits, de sciences ou de beaux-arts, situés dans une grande métropole ou à la campagne, dépendant ou non des pouvoirs publics. S'il est fort probable que le modèle du musée connaisse des transformations dans un futur proche, il semble tout aussi évident que celles-ci ne s'appliqueront pas de manière uniforme, et que par ailleurs, certaines d'entre elles ne sont pas encore connues. Il nous reste à les penser et à les construire collectivement. 


\section{NOTES}

1. National Intelligence Council, Global Trends 2030 : Alternative worlds, Washington, NIC, 2012. Disponible sur Internet : http://info.publicintelligence.net/GlobalTrends2030.pdf

2. Ministère de la Culture et de la Communications, Culture \& Médias 2030. Prospective de politiques culturelles. Paris : La Documentation française, 2011. Disponible sur Internet : www.culturemedias2030.culture.gouv.fr/

3. Ces trois rapports sont disponibles sur le site du Centre pour l'avenir des musées : www.aamus.org/resources/center-for-the-future-of-museums

4. Nederlandse Museumvereniging, Agenda 2026. Study on the Future of the Dutch Museum Sector, Amsterdam, september 2010. Disponible sur Internet : www.museumvereniging.nl/ LinkClick.aspx?fileticket=jEC-ghnfc3A\%3D\&tabid=244

5. Museums Association, Museums 2020 Discussion Paper, London, Museums Association, 2012. Disponible sur Internet : www.museumsassociation.org/download?id=806530.

6. Van Mensch, P. and L. New Trends in Museology. Celsje : Museum of Recent History, 2011.

7. Par ailleurs, si presque tous les rapports sont structurés à partir des principaux facteurs de changement, celui utilisé par la Museums Association se fonde sur un scénario du futur, construit à partir de ces transformations.

8. Black, G. Transforming Museums in the Twenty-first Century. London : Routledge, 2012.

\section{RÉSUMÉS}

La prospective dont il question ici concerne l'avenir des institutions muséales analysé à la lueur de différents facteurs (démographie, mondialisation, économie, innovation technologique, éducation, muséologie), du rôle joué dans cette réflexion par des associations professionnelles de musées, des principales tendances qui vont influencer les musées à l'horizon 2025-2030 et la manière possible pour eux de s'adapter et de se préparer aux mutations futures.

\section{AUTEUR}

\section{FRANÇOIS MAIRESSE}

François Mairesse est professeur à l'université Paris 3-Sorbonne nouvelle, CERLIS, ICCA.

francois.mairesse@univ-paris3.fr 